\title{
Parkinson's Disease and Early Stages of Progressive Supranuclear Palsy: A Neurologieal Mimicry
}

\section{JER}

\author{
Robin George', Vinod Krishnan'2, Biju Mohan', Pavan Maniyar ${ }^{3}$ \\ Departments of ' Medicine and ${ }^{3}$ Radiology, National Hospital, Calicut, Kerala; ${ }^{2}$ Department \\ of Medicine, KMCT Medical College, Calicut, Kerala, India.
}

Abstract:

Progressive supranuclear palsy (PSP) is not a rare condition, but because of its increasingly recognized clinical diversity, it may be difficult to diagnose, especially in a resource poor setting where imaging modalities may not be available. As a result, progressive supranuclear palsy is often misdiagnosed as Parkinson's disease (PD). In this case report, we would like to highlight the importance of brain Magnetic Resonance Imaging (MRI) in diagnosing early stage of progressive supranuclear palsy in a patient who was being treated as Parkinson's disease.

Key words: Brain Stem, Magnetic Resonance Imaging, Parkinson's Disease, Progressive Supranuclear Palsy.

\section{Introduction}

PSP is a sporadic neurodegenerative disorder of unknown etiology which is characterized by akinetic rigid parkinsonism, dizziness, unsteadiness, slowness, falls, and pseudobulbar dysarthria. Tremor is distinctly uncommon [1]. In 1963, John Clifford Richardson described this "unusual syndrome" and his collaborators John Steele and Jerzy Olszewski identified consistent pathological findings that were eventually used to establish this new nosological condition which was also called Steele-RichardsonOlszewski syndrome [2]. Pathologically, this condition is defined by the accumulation of tau protein and neuropil threads, mainly in the subthalamic nucleus, pallidum, red nucleus, substantia nigra, pontine tegmentum, striatum, medulla, oculomotor nucleus, and dentate nucleus
[3]. Moreover, histopathological findings similar to PSP are seen in patients with postencephalitic parkinsonism (PEP) and the atypical parkinsonian syndromes of Guam and Guadeloupe, thus making the pathological diagnosis of PSP complicated $[4,5]$. The National Institute of Neurological Disorders and Stroke (NINDS) has outlined SPSP criteria for defining probable and possible PSP, and definite PSP requiring histopathology.

Despite all these advances in our understanding of the disease process, the accurate diagnosis of PSP depends on clinical acumen, as there are no reliable diagnostic biomarkers. However, clinical differentiation of PSP from PD and multiplesystem atrophy of Parkinson can be difficult,

\section{Corresponding Author: Dr. Robin George}

Email: drrobingeorgempl@gmail.com

Received: June 30, 2015 | Accepted: August 24, 2015 | Published Online: September 5, 2015

This is an Open Access article distributed under the terms of the Creative Commons Attribution License (creativecommons.org/licenses/by/3.0)

Conflict of interest: None declared | Source of funding: Nil | DOl: http://dx.doi.org/10.17659/01.2015.0097 
particularly in early stages of the disease. MRI studies on PSP have shown atrophy of the midbrain and pons in mid-sagittal sections, hence aiding in its diagnosis [6]. The goal of this case report is to highlight the usefulness of MRI brain in diagnosing PSP, especially in its early stages, and preventing its misdiagnosis as PD.

\section{Case Report}

Our patient is a 68 year old male, a retired office worker, who presented with history of fall, which he says was due to dizziness. There was no history of loss of consciousness/seizures/weakness/trauma. Patient is a hypertensive on regular medications (losartan and amlodipine).

Detailed history revealed that the patient has been symptomatic since past 4 months and his complaints constituted of rest tremors, mild slurring of speech and bradykinesia. He also gives history of dementia, irritability and decreased sleep. On the basis of these complaints, the patient was diagnosed to have PD and was started on levodopa / carbidopa by an outside clinician. No brain imaging was done. He has been taking this medication for past 4 months, but there was no improvement in his symptoms. About 2 months ago, patient had a similar fall, following which he had a fracture of right humerus and internal fixation was done. He had undergone TURP operation andbilateral inguinal hernial repair 2 years and 8 years ago respectively.

Examination of the patient revealed a fully conscious male (mini-mental assessment normal), with normal vitals, who had bradykinesia, tremors and slurred monotonous speech. Motor system showed cogwheel rigidity and cranial nerve examination revealed defective downward gaze. Glabellar tap reflex was present but applause sign was absent. His sensory system was normal and cerebellar signs were absent. He had an erect posture with retropulsive gait.
Though the patient's initial presentation may correspond to PD, the presence of downward gaze difficulty, history of fall and lack of response to levodopa/carbidopa raised the suspicion of PSP. Hence, MRI brain was taken which showed atrophy of midbrain [Fig. 1,2]. The pontine area, midbrain area and pons midbrain ratio were $4.22 \mathrm{~cm}^{2}$, $0.5 \mathrm{~cm}^{2}$ and 8.44 respectively. The width of superior cerebellar peduncle was $40 \mathrm{~mm}$ and middle cerebellar peduncle $160 \mathrm{~mm}$.

\section{Patient was continued on levodopa/} carbidopa. Zolpidem was started to ameliorate bradykinesia and rigidity. However, the limitations in treatment and observing the decline of these patients are a frustrating ordeal.

\section{Discussion}

PSP is not a rare disease but the dearth of published epidemiological studies makes it difficult

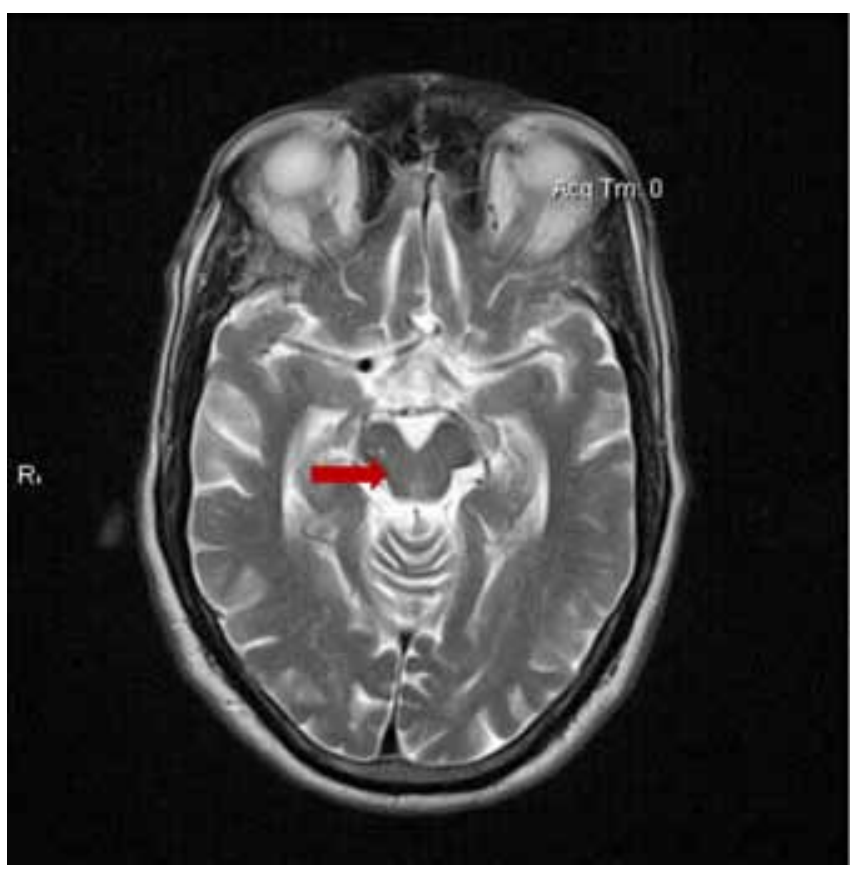

Fig.1: Brain MRI of the patient showing measurement of the midbrain and pons and their values. 
to determine the incidence and prevalence rates. The estimated annual incidence was about 0.3-1.1 cases per 100,000 persons [7]. Genetically, patients with PSP frequently show $\mathrm{H} 1$ halotype and $\mathrm{HI} / \mathrm{HI}$ genotype [8]. PSP affects both sexes, with a slight male preponderance. The disease has a significantly shorter mean survival than PD [9]. Macroscopic examination of the brain shows midbrain atrophy and substantia nigra depigmentation, sometimes with mild frontal lobe atrophy while microscopic examination shows neuronal loss, gliosis, neurofibrillary tangles, and neuropil threads in the basal ganglia and brainstem. PSP is characterizedby hyperphosphorylated tau protein aggregates [10].

The onset is typically in the sixth decade (range 45 to 75 years) [ 11 ]. The clinical hallmarks of PSP are defined as vertical gaze palsy, axial rigidity, pseudobulbar palsy and cognitive impairment [12]. Since 1964, there has been an evolution in these criteria with inclusion of falls within the first year of illness and, more specifically, supranuclear gaze palsy [13]. The most common early symptom is unsteady gait and unexplained falling without loss of consciousness. The other characteristic symptoms may take a year or longer to develop. Difficulty in voluntary vertical eye movements, often downward, and later impairment of voluntary saccades in all directions are characteristic. There is no gait ataxia but patients tend to lean and fall backward. There is also gradual stiffening and extension of neck. The patient's face acquires a staring, worried look with furrowed brow. The face becomes less expressive, speech is slurred, the mouth tends to be held open and swallowing becomes difficult. Many patients may complaint of sleep disturbances [11].

PSP gets progressively worse but is not itself directly life-threatening. The most common complications are choking and pneumonia, head injury, and fractures caused by falls. There is currently no effective treatment for PSP. In some

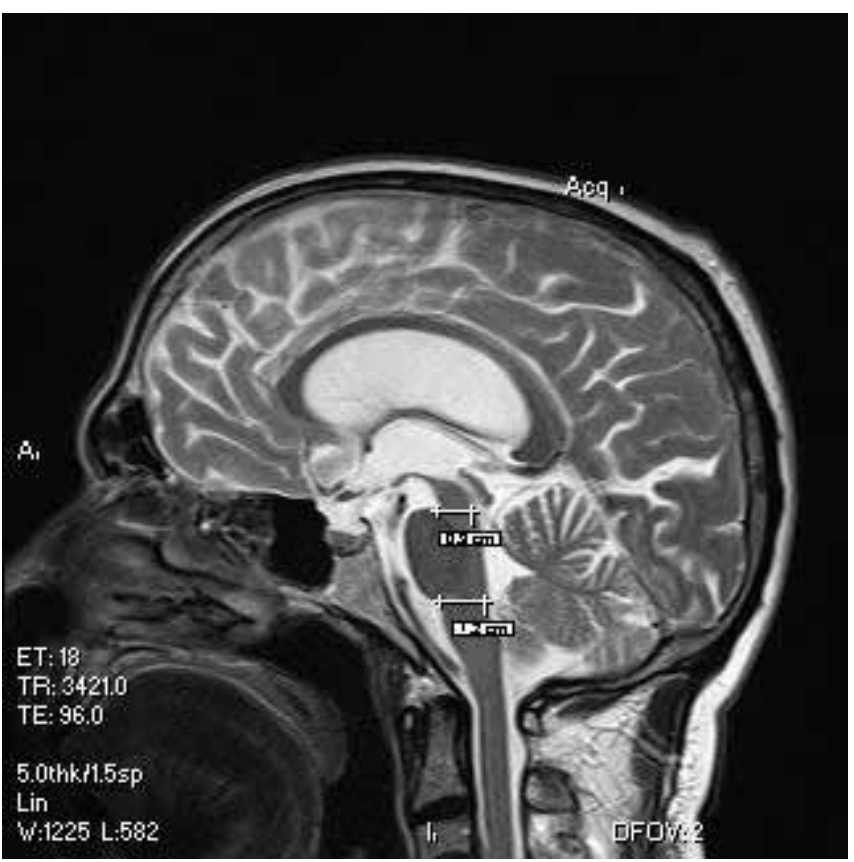

Fig.2: Brain MRI of the patient showing atrophy of midbrain.

individuals the slowness, stiffness, and balance problems of PSP may respond to anti-parkinsonian agents such as levodopa, but the effect is usually temporary. Excessive eye closing can respond to botulinum injections. Zolpidem has been found to ameliorate bradykinesia and rigidity. Non-drug treatment for PSP include the use of weighted walking aids because of their tendency to fall backward and bifocals or special glasses called prisms are sometimes prescribed as remedy for difficulty of looking down. A gastrostomy (or a jejunostomy) may be necessary when there are swallowing disturbances or the definite risk of severe choking. Formal physical therapy is of no proven benefit in PSP, but certain exercises can be done to keep the joints limber. Deep brain stimulation and other surgical procedures used in individuals with Parkinson's disease have not been proven effective in PSP. Autologous adipose tissuederived mesenchymal stem cells transplantation show promising results in delaying the progression of PSP [14]. 
PD is the most common form of progressive neurodegenerative disorders characterized by bradykinesia, rest tremor, muscular rigidity, shuffling gait, and flexed posture. PD can be accompanied by autonomic, sensory, sleep, cognitive, and psychiatric disturbances. A diagnosis of PD should satisfy at least two of the three cardinal signs- rest tremor, rigidity, and bradykinesia. Parkinsonism results from a reduction of dopaminergic transmission within the basal ganglia. Although the vast majority of cases appear to be sporadic, there is increasing evidence that genetic factors play an important role in many forms of PD. PARK1, PARK4, and PARK5 lead to an autosomal dominant form of PD while PARK2 and PARK7 lead to autosomal recessive disorders. Pathologically, there is mild frontal atrophy with loss of the normal dark melanin pigment of the midbrain and microscopically there is degeneration of the dopaminergic cells with the presence of Lewy bodies in the remaining neurons and processes of the substantianigra pars compacta. Lewy bodies have a high concentration of $\alpha$-synuclein and its mutation can cause familial PD. Levodopa remains the most effective treatment for PD as it significantly improves motor symptoms and increases quality of life [1].

Accurate clinical differentiation of PSP from PD may be difficult, particularly in the early stages of the disease. MRI brain studies on PSP emphasized the utility of mid-sagittal MRI in its diagnosis. The range of midbarin area, pontine area and midbrain pons ratio was lower in PSP as compared to PD [15]. A study by $\mathrm{Oba}$ et al. concluded that midbrain area $<70 \mathrm{~mm}$ was strongly suggestive of PSP, whereas midbrain pons ratio $<0.15$ excludes the diagnosis of PD. The authors also proposed the "penguin silhovette" sign which was present in all patients with PSP on the mid-sagittal MRI [16]. A "humming bird sign" was alluded by Kato et al. based on midbrain pons ratio from MRI of PSP patients [17]. Atrophy of superior cerebellar peduncle is also evident in these patients [18]. Mid-sagittal MRI images provide a clearer view of atrophy of the midbrain and pons and dilatation of the cisterns than axial images [19]. The reduction of the midbrain area, pontine area and midbrain pontine ratio significantly correlated with the duration and staging of the disease and manifestations of disease severity [20].

The case being reported emphasizes the usefulness of $M R I$ brain in reinforcing clinical suspicion of PSP, especially in early stages. However, the mainstay of diagnosis continues to be the criteria outlined by NINDS [21]. This is due to the fact that there is no sufficient data to correlate MRI findings to pathological data. But in early stages of PSP, where there are chances of misdiagnosis, MRI brain can offer substantial benefits.

\section{Conclusion}

The frequency of misdiagnosis of PD is still 10\%$25 \%$. Neurological assessment (development of early imbalance, falls, and characteristic abnormalities of vertical gaze) remains as the mainstay for diagnosis of PSP. However, the condition may be misdiagnosed as PD, especially in its early stages. As PSP is not a rare condition, and in order to prevent its misdiagnosis as $P D$, this case report highlights the utility of $M R I$ brain in assisting the diagnosis of PSP. However, the very fact of limitations of treatment and observing the decline of these patients becomes a frustrating ordeal to both the clinician as well as the family members and it finally raises the question "What next after making this diagnosis?"

\section{References}

1. Mahon RD, Jorge LJ. Parkinson's Disease and Other Extrapyramidal Movement Disorders. Harrison's Principles of Internal Medicine. 17 th edition. Vol 2, Chap 366, pp. 2559.

2. Steele JC, Richardson JC, Olszewski J. Progressive supranuclear palsy. A heterogeneous degeneration involving the brain stem, basal 
ganglia and cerebellum with vertical supranuclear gaze and pseudobulbar palsy, nuchal dystonia and dementia. Arch Neurol.1964;10:333-359.

3. Hauw JJ, Daniel SE, Dickson D, Horoupian DS, Jellinger K, Lantos PL, et al. Preliminary NINDS neuropathologic criteria for Steele-RichardsonOlszewski syndrome (progressive supranuclear palsy). Neurology.1994; 44:2015-519.

4. Yamazaki M, Makifuchi T, Chen KM, Mori O, Katayama $Y$, Takahashi $H$, et al. Progressive supranuclear palsy on Guam. Acta Neuropathol. 2001;102:510-514.

5. Oyanagi K, Chen KM, Craig UK, Yamazaki $M$, Perl DP. Parkinsonism, dementia and vertical gaze palsy in a Guamanian with atypical neuroglial degeneration. Acta Neuropathol. 2000;99:73-80.

6. Cosottini $M$, Ceravolo R, Faggioni L, Lazzarotti $G$, Michelassi MC, Bonuccelli $U$, et al. Assessment of midbrain atrophy in patients with progressive supranuclear palsy with routine magnetic resonance imaging. Acta Neurol Scand. 2007; $116(1): 37-42$.

7. Bower JH, Maraganore DM, McDonnell SK, Rocca WA. Incidence of progressive supranuclear palsy and multiple system atrophy in Olmsted County, Minnesota,1976 to 1990. Neurology.1997;49:1284-1288.

8. Bennett $P$, Bonifati V, Bonuccelli U, Colosimo C, De Mari $M$, Fabbrini $G$, et al. Direct genetic evidence for involvement of tau in progressive supranuclear palsy. European Study Group on Atypical Parkinsonism Consortium. Neurology. 1998:51:982-985.

9. Golbe LI. Progressive supranuclear palsy. In: Beal $M$ Flint, Lang $A E$, Ludolph $A$, edsNeurodegenerative diseases. Cambridge: Cambridge University Press, 2005;663-681.

10. Ramakrishnan TCR, Ramesh S, Senthil Velkumar T. Facial Diplegia: A Misleading GBS Variant. Journal of Case Reports. 2013;3(2):267-271.

11. Alan HR, Martin AS. Degenerative Diseases of Nervous System. Adams and Victor's Principles of Neurology. $9^{\text {th }}$ edition. Chapter 39; pp 10461048.
12. Duvoisin RC. Clinical diagnosis. In: Litvan I, Agid $Y$, editors. Progressive Supranuclear Palsy: Clinical and Research Approaches. New York: Oxford University Press; 1992. pp. 15-33.

13. Lubarsky $M$, Juncos JL. Progressive supranuclear palsy: A current review. Neurologist. 2008; 1 4:7988.

14. Soo Won Choi, Kwon Byong Park, Sang Kyu Woo, Sung Keun Kang, Jeong Chan Ra. Treatment of progressive supranuclear palsy with autologous adipose tissue-derived mesenchymal stem cells: a case report. Journal of Medical Case Reports. $2014 ; 8: 87$

15. Emam AT, El-Sayed EA, Awad FM. Progressive Supranuclear Palsy: Brainstem Measurement and its Clinical Implication. Egypt J NeurolPsychiatNeurosurg. 2010;47:355.

16. Oba H, Yagishita A, Terada H, Barkovich AJ, Kutomi K, Yamauchi T, et al. New and reliable MRI diagnosis for progressive supranuclear palsy. Neurology. 2005;64:2050-2055.

17. Kato N, Arai K, Hattori T. Study of the rostral midbrain atrophy in progressive supranuclear palsy. J Neurol Sci. 2003;210:57-60.

18. Tsuboi Y, Slowinski J, Josephs KA, Honer WG, Wszolek ZK, Dickson DW. Atrophy of superior cerebellar peduncle in progressive supranuclear palsy. Neurology. 2003;60:1766-1769.

19. Wszolek ZK, Slowinski J, Imamura A, Tsuboi Y, Broderrichk DF. New and reliable MRI diagnosis for progressive supranuclear palsy. Neurology. 2006;66(5):781.

20. Halliday GM, Hardman CD, Cordato NJ, Hely MA, Morris JG. A role for the substantia nigra pars retriculata in the gaze palsy of progressive supranuclear palsy. Brain. 2000;1 23:724-732.

21. Litvan I, Agid, Y, Calne D, Campbell G, Dubois B, Duvoisin RC, et al. Clinical research criteria for the diagnosis of progressive supranuclear palsy (Steele-Richardson-Olszewski syndrome): Report of the NINDS-SPSP international workshop. Neurology. 1996;47(1):1-9. 